\title{
ESTUDO DO COMPORTAMENTO FLUIDODINÂMICO DE UM LEITO VIBROFLUIDIZADO OPERANDO COM DIFERENTES DIÂMETROS DE PARTÍCULAS
}

\author{
A. B. S. $\operatorname{COSTA}^{1}$, F. B. $\operatorname{FREIRE}^{1}$, J. T. FREIRE ${ }^{1 *}$ \\ ${ }^{1}$ Universidade Federal de São Carlos, Departamento de Engenharia Química \\ e-mail: freire@ufscar.br
}

\begin{abstract}
RESUMO
Analisar o comportamento fluidodinâmico de um equipamento é uma etapa importante para auxiliar na compreensão dos fenômenos presentes durante o processo de secagem. Por isso que o objetivo deste trabalho consiste em investigar o comportamento dinâmico do leito vibrofluidizado de forma mais detalhada, quando submetido a diferentes intensidades de vibração utilizando as amplitudes (A) de $0,015 \mathrm{~m}$ e $0,003 \mathrm{~m}$, associadas a diferentes frequências (f), que variaram de 100 em $100 \mathrm{rpm}$, quantificadas de acordo com o número adimensional de vibração $(\Gamma)$ e operando com esferas de vidro de $1,19 \mathrm{~mm}$ e de $2,19 \mathrm{~mm}$ de diâmetro. A finalidade foi verificar a influência que estas variáveis exerceram na queda de pressão $(\Delta \mathrm{p})$ máxima do leito, na velocidade de mínima fluidização $\left(U_{\mathrm{mf}}\right)$ e no desvio padrão da queda de pressão $(\delta p)$. Os resultados mostraram que o comportamento fluidodinâmico foi dependente das combinações entre A, f, e do tamanho da partícula trabalhada. Além de mostrar que $\Gamma$ não deve ser usado para caracterizar o leito.
\end{abstract}

\section{INTRODUÇÃO}

O leito vibrofluidizado é um equipamento que surgiu da necessidade de fluidizar partículas grosseiras e materiais com características coesivas e pastosas, uma vez que, o leito fluidizado convencional (LFC) apresenta uma boa fluidização apenas para as partículas que estão classificadas no grupo A e B de Geldart (1974) (KUNII E LEVENSPIEL, 1991). O seu diferencial está na vibração imposta ao sistema com a finalidade de promover um efetivo contato entre a fase líquida e a fase sólida, que o LFC muitas vezes não consegue proporcionar para alguns tipos de partículas.

Esta vibração desenvolvida no equipamento foi quantificada de diversas maneiras, no entanto, a equação que frequentemente é utilizada pela maioria dos trabalhos presentes na literatura, é definida como o número adimensional de vibração e está apresentada na Equação 1.

$$
\Gamma=\frac{A(2 \pi f)}{g}
$$

onde A é a amplitude, f a frequência vibracional e $\mathrm{g}$ a aceleração gravitacional.

Com o transcorrer dos anos, este tipo de equipamento tornou-se conveniente em diferentes industriais, podendo ser aplicado em diversos processos como, secagem de materiais granulares (SILVA-MORIS e ROCHA, 2006), na secagem de materiais pastosos (MEILI ET AL, 2010), no recobrimento de partículas (NUNES ET AL. 2012) e na granulação (COSTA ET $A L$. 2011).

Para o processo de secagem de pasta, tem-se a necessidade do uso de material inerte, atuando como suporte para a pasta, 
para proporcionar um maior contato da pasta com o fluido e também como uma fonte extra de calor (FREIRE ET AL., 2012). Desta técnica, existem vários leitos móveis que são promissores para realizarem este tipo de processo, entretanto, o leito vibrofluidizado tem apresentado destaque, pois a vibração desenvolvida neste equipamento, em conjunto com o escoamento do fluido, contribuem com a movimentação do material inerte e facilitam a fludização, além de desenvolver efetivas taxas de transferência de calor e massa, e reduzir o consumo de energia, com baixos valores de velocidade de mínima fluidização e da queda de pressão no interior do leito, quando comparados aos leitos fluidizados convencionais (GUPTA E MUJUMDAR, 1980; KUNII E LEVENSPIL, 1991).

Para compreender os mecanismos e fenômenos envolvidos durante a secagem de pasta no leito vibrofluidizado, é importante caracterizar o comportamento dinâmico do leito diante dos diferentes tipos de materiais inertes (BRATU E JINESCU 1971; DALEFFE ET AL., 2008; SILVA-MORIS e COSTA 2006 e GUPTA e MUJUMDAR 1980). Bem como das variáveis operacionais envolvidas no processo. Daleffe, et al. (2005), por exemplo, verificaram que o $\Gamma$ de mesmo valor apresentou comportamentos fluidodinâmicos distintos. Meili et al. (2010) comprovaram que o $\Gamma$ não pode ser usado como parâmetro único para caracterizar a dinâmica do leito, destacando que deve fornecê-lo juntamente com $\mathrm{A}$ ou com $\mathrm{f}$ para demonstrar o efeito da vibração. No entanto, Nunes et al. (2012), verificaram que uma mesma amplitude combinada a duas frequências diferentes, resultaram em medidas de queda de pressão bem próximas.

Com o intuito de dar continuidade aos estudos sobre o comportamento dinâmico do leito vibrofluidizado, este trabalho tem a finalidade de estudar a fluidodinâmica do leito vibrofluidizado operando com duas esferas de vidro de diâmetros diferentes
$(1,19 \mathrm{~mm}$ e $2,19 \mathrm{~mm})$, realizando uma investigação mais detalhada da influência que a amplitude, a frequência de vibração e o número adimensional de vibração, podem exercer nos parâmetros, velocidade de mínima fluidização $\left(\mathrm{U}_{\mathrm{mf}}\right)$, queda de pressão do leito $(\Delta \mathrm{p})$ e desvio padrão de queda de pressão $(\delta \mathrm{p})$.

\section{MATERIAS MÉTODOS}

\subsection{Unidade experimental e materiais}

Para a realização dos experimentos foi utilizado um leito vibrofluidizado de acordo com a unidade experimental apresentada na Figura 1.

A unidade experimental do leito é constituída de um soprador (1), um trocador de calor (2) ligado a um controlador de temperatura (17), um resfriado (3), uma câmara de vidro (11) e um ciclone (19). Para o ajuste e controle da vazão que escoa no equipamento foi usada uma placa de orifício (4) conectada a um transdutor de pressão (6). Além da placa de orifício, a base do leito também foi conectada a um transdutor de pressão (6) para que fosse obtida a tomada da pressão no leito. Todos os transdutores e termopares estavam conectados a uma placa de aquisição de dados instalada em um computador (7). O sistema de aquisição foi desenvolvido utilizando o Software Labview.

Como material inerte, optou-se por empregar dois tipos de esferas de vidro, as quais apresentam o diâmetro médio de Sauter de $2,19 \mathrm{~mm}$ e $1,19 \mathrm{~mm}$, e massa específica aparente de $2500 \mathrm{~kg} / \mathrm{m}^{3}$. Os ensaios foram desenvolvidos usando um massa de material inerte igual a $1,5 \mathrm{~kg}$, que corresponde a uma altura de leito estático com aproximadamente $9 \mathrm{~cm}$.

\subsection{Procedimento experimental.}

Os experimentos foram conduzidos de acordo com as condições experimentais apresentadas e organizadas na Tabela 1 , as 
quais foram selecionadas após serem realizados alguns testes experimentais para a determinação das frequências a serem trabalhadas, que mantivessem a estabilidade estrutural do equipamento, uma vez que preferiu-se manter as duas amplitudes trabalhadas por Meili (2009), 0,003m e $0,015 \mathrm{~m}$.

Também foram realizados experimentos para $\Gamma=0$, que corresponde ao leito fluidizado, e também para o leito vibrofluidizado em que o $\Gamma=1$, com duas combinações diferentes de amplitude e frequência $(A=0,003 \mathrm{~m}$ e $550 \mathrm{rpm}$; e $A=0,015 \mathrm{~m}$ e $245 \mathrm{rpm}$ ). Para estas últimas condições foram feitas três repetições, para cada partícula, a fim de confirmar a confiabilidade dos dados trabalhados. Este valor de $\Gamma$ foi selecionado uma vez que corresponde à condição de transição do comportamento dinâmico do leito com agitação, separando os leitos agitados que tendem a compactar o material particulado, dos leitos mais soltos.

Tabela 1- Condições experimentais.

\begin{tabular}{ccc}
\hline $\begin{array}{c}\text { Amplitude } \\
(\mathrm{m})\end{array}$ & $\begin{array}{c}\text { Frequência } \\
(\mathrm{RPM})\end{array}$ & $\begin{array}{c}\text { Adimensional } \\
\text { de vibração } \\
(\Gamma)\end{array}$ \\
\hline 0,003 & 200 & 0,13 \\
0,003 & 300 & 0,29 \\
0,003 & 400 & 0,53 \\
0,003 & 500 & 0,84 \\
0,003 & 600 & 1,2 \\
0,003 & 700 & 1,65 \\
0,003 & 800 & 2,15 \\
0,003 & 900 & 2,7 \\
0,003 & 1000 & 3,36 \\
0,003 & 1100 & 4,06 \\
0,015 & 200 & 0,65 \\
0,015 & 300 & 1,48 \\
0,015 & 400 & 2,69 \\
0,015 & 500 & 4,2 \\
0,015 & 600 & 6,04 \\
\hline
\end{tabular}

Fonte: Acervo pessoal.

Figura 1- Esquema da unidade experimental.

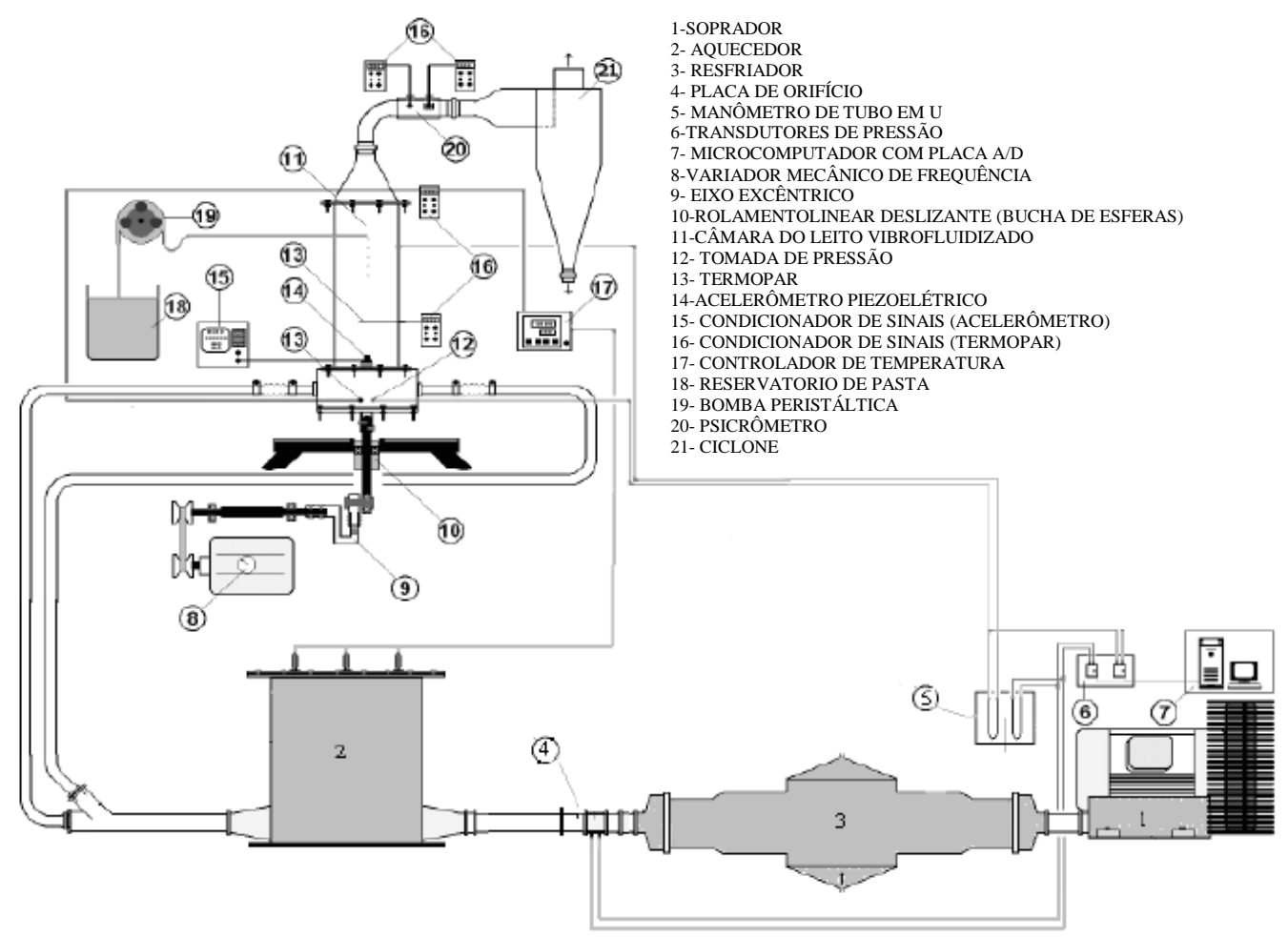

Fonte: Meili 2009. 
Para obter as curvas fluidodinâmicas, os experimentos foram conduzidos ajustando as condições de amplitude e frequência no equipamento. Em seguida, o leito era mantido em regime fluidizado até atingir a estabilização das condições operacionais. Atingida a estabilidade, iniciou-se a obtenção dos dados de queda de pressão $(\Delta p)$ em função da velocidade superficial do ar (Us). Essas medidas foram efetuadas para velocidade do ar decrescente, em que reduziase esta variável para um novo valor e aguardava-se reestabelecer o equilíbrio, metodologia clássica apresentada por Bratu e Jinescu (1971). No equilíbrio, o sistema de aquisição de dados também fornecia os valores de desvio padrão de queda de pressão, obtidos de 1024 pontos, coletados a cada 30 segundos, para auxiliar nas análises da dinâmica do leito, conforme foi proposto por Garim (1998). Este procedimento foi realizado para todas as condições apresentadas na Tabela 1, onde uma nova situação era estabelecida e efetuava-se novamente a redução da velocidade do ar, para a obtenção das novas medidas.

Para determinar a velocidade de mínima fluidização de cada curva fluidodinâmica obtida no leito vibrofluidizado, usou-se a metodologia empregada no leito fluidizado convencional, que corresponde à inserção de duas retas tangentes na curva de queda de pressão em função da velocidade superficial do ar, sendo que uma reta tangente na região de leito fixo e a outra reta tangente na região de fluidização (KUNII E LEVENSPIL 1991).

\section{RESULTADOS E DISCUSSÕES.}

Inicialmente serão analisadas a reprodutibilidade dos dados apresentados nas Figuras 2 e 3 , que mostram as curvas de $\Delta \mathrm{p}$ e $\delta \mathrm{p}$ em função da velocidade superficial do ar, para as esferas com os diâmetros de $1,19 \mathrm{~mm}$ e $2,19 \mathrm{~mm}$, respectivamente, para o $\Gamma=1,0 \mathrm{em}$ duas combinações de A e f.
Figura 2- Queda de pressão e desvio padrão de queda de pressão em função da velocidade superficial do ar, para as esferas de $1,19 \mathrm{~mm}$ de diâmetro e $\Gamma=1 \mathrm{com}$, (a) $\mathrm{f}=550 \mathrm{rpm}$ e $\mathrm{A}=3 \mathrm{~mm}$, e (b) $\mathrm{f}=$ de $245 \mathrm{rpm}$ e $\mathrm{A}=15 \mathrm{~mm}$.
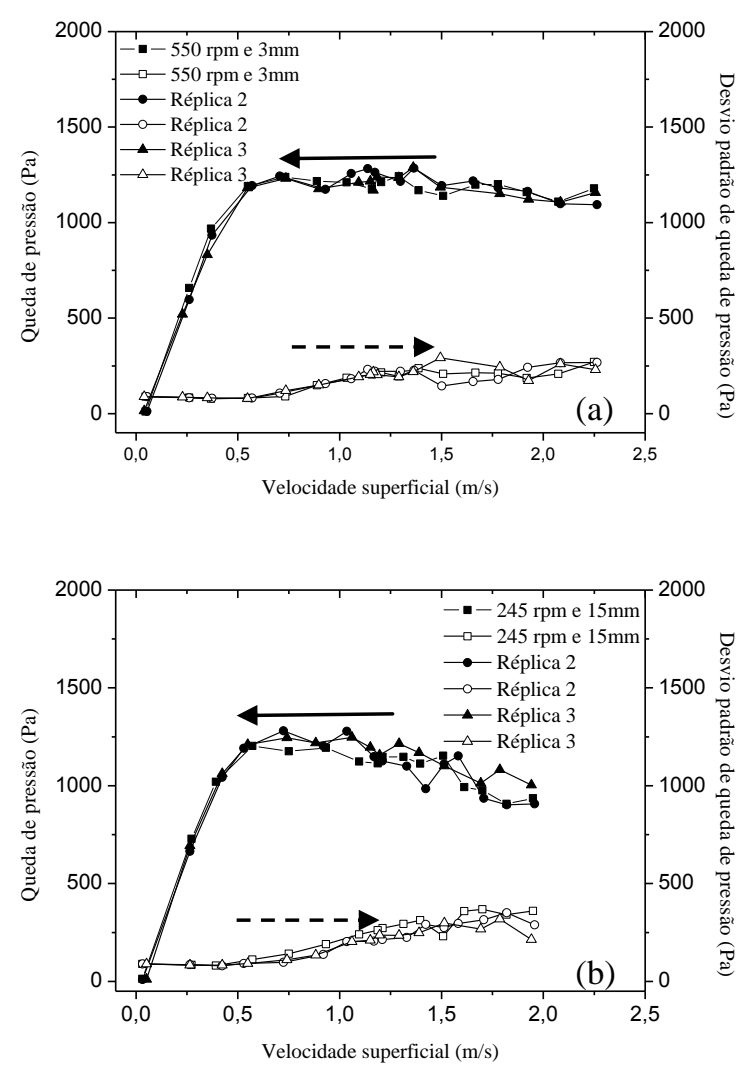

Fonte: Acervo pessoal.

Na Figura 2a verificou-se, que para o leito com esferas de $1,19 \mathrm{~mm}$ de diâmetro, os dados de queda de pressão obtidos para a amplitude de $0,003 \mathrm{~m}$ apresentaram uma boa reprodutibilidade nas medidas, tanto para a região de leito fixo, quanto para a região de fluidização, mostrando pouca discrepância entre os pontos. No entanto, quando foi analisada a Figura $2 b$, referente aos experimentos realizados com amplitude de $0,015 \mathrm{~m}$, verificou-se que os dados também apresentaram bons resultados, mostrando apenas que a região de fluidização possui uma maior disparidade entres as medidas. Esta dificuldade encontrada em reproduzir a região de fluidização, para esta amplitude, pode estar relacionada com as forças que estavam 
presentes durante $\mathrm{o}$ processo e que promoveram um arranjo desordenado das esferas vidro.

Figura 3- Queda de pressão e desvio padrão de queda de pressão em função da velocidade superficial do ar, para as esferas de $2,19 \mathrm{~mm}$ de diâmetro e $\Gamma=1 \mathrm{com}$, (a) $\mathrm{f}=550 \mathrm{rpm}$ e $\mathrm{A}=3 \mathrm{~mm}$, e (b) $\mathrm{f}=$ de $245 \mathrm{rpm}$ e $\mathrm{A}=15 \mathrm{~mm}$.
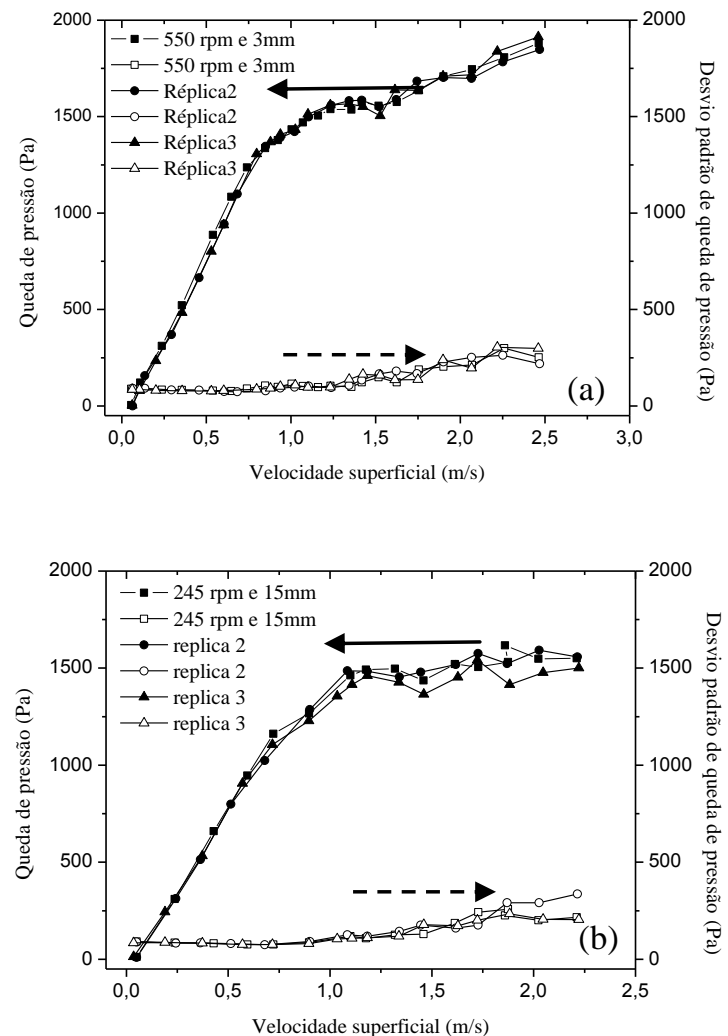

Fonte: Acervo pessoal.

Na Figura 3a, verificou-se que para o leito particulado com esferas de $2,19 \mathrm{~mm}$ de diâmetro, os dados de queda de pressão obtidos para a amplitude de 0,003m, apresentaram boa reprodutibilidade nas medidas, com pouca disparidade entre os pontos, tanto para a região do leito fixo, quanto para a região de fluidização. Ao analisar a Figura $3 b$, verificou-se que os valores de queda de pressão obtidos para a amplitude de $0,015 \mathrm{~m}$ também apresentaram uma boa reprodutibilidade dos dados, com a região de fluidização tendo uma maior discrepância entre as medidas, porém uma diferença aparentemente menor do que foi observado para a esfera de $1,19 \mathrm{~mm}$.

A Figura 4 apresenta os dados de $\Delta \mathrm{p}$ e do $\delta \mathrm{p}$ em função da velocidade superficial do ar, obtidos no leito fluidizado convencional para os dois tamanhos de esferas de vidro.

Figura 4- Queda de pressão e desvio padrão de queda de pressão em função da velocidade superficial do ar, para o leito com $\Gamma=0$.

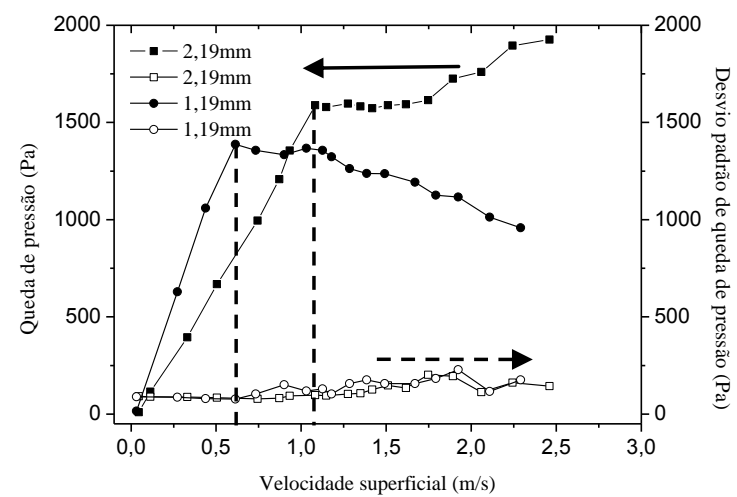

Fonte: Acervo pessoal.

Os resultados encontrados na Figura 4 apresentaram, para as duas esferas, curvas com o comportamento característico do leito fluidizado convencional, ficando evidente a transição do leito fixo para o leito fluidizado. Como usou-se esferas de tamanhos diferentes, foi constatado que esta transição aconteceu em diferentes velocidades de mínima fluidização. Para as esferas com 1,19mm de diâmetro, a velocidade de mínima fluidização foi de aproximadamente $0,6 \mathrm{~m} / \mathrm{s}$. As esferas com 2,19mm de diâmetro, a velocidade mínima fluidização foi de aproximadamente $1,1 \mathrm{~m} / \mathrm{s}$. Como são esferas de vidro classificadas no grupo C $(1,19 \mathrm{~mm})$ e no grupo D $(2,19 \mathrm{~mm})$ de Geldart (1975), as forças interparticulares também deixam de ser significativas no processo. Seville, Willett e Knight (2000) afirmaram que esferas com diâmetros superiores a $1 \mathrm{~mm}$, as forças interparticulares deixam de ser significantes no processo, sendo a força gravitacional 
apenas a força resultante. Com isso os valores encontrados de velocidade de mínima fluidização estão relacionados apenas com o peso de cada partícula, pois ao aumentar o diâmetro da esfera, aumentou-se a massa da mesma e necessitou de uma velocidade maior para fazer com que o conjunto de partículas alcançasse a fludização.

Ao analisar as curvas obtidas do desvio padrão de queda de pressão, verificouse um comportamento semelhante para ambas as esferas de vidro, em que na região de leito fixo os desvios foram muito baixos, bem próximos de zero, e na região de fluidização incipiente, os valores destes desvios tiveram um pequeno aumento, indicando que houve um aumento nas oscilações de queda de pressão. Uma vez que as esferas de vidro têm diâmetros diferentes e apresentaram diferentes, velocidades de mínima fluidização e quedas de pressão máxima, os valores de desvio padrão de queda de pressão não apresentaram muita diferença, pois a disparidades entre as medidas foi bem pouca.

Nas Figuras 5 a 8 serão apresentadas o comportamento dinâmico do leito particulado diante de diferentes intensidades de agitação e tamanhos das esferas de vidro. A Figura 5 mostra as curvas de $\Delta p$ e $\delta p$ em função da velocidade superficial do ar, obtidos no leito com partículas com 1,19mm de diâmetro, diante de uma agitação com amplitude de $0,003 \mathrm{~m}$ combinada com diferentes frequências. Nesta figura, foi observado que os valores máximos da queda de pressão foram bem próximos uns dos outros e variaram de $1292 \mathrm{~Pa}$, quando a amplitude foi combinada com a frequência de $200 \mathrm{rpm}$, e $1223 \mathrm{~Pa}$, quando foi combinada com a frequência de $1100 \mathrm{rpm}$. Como a amplitude foi pequena, a agitação promoveu semelhantes estruturas e porosidades do leito de partículas durante o processo e por isso valores de queda de pressão bem próximos.
Figura 5- Queda de pressão e desvio padrão de queda de pressão em função da velocidade superficial do ar, para as esferas de vidro com $1,19 \mathrm{~mm}$ de diâmetro e $\mathrm{A}=0,003 \mathrm{~m}$ combinada com diferentes frequências.

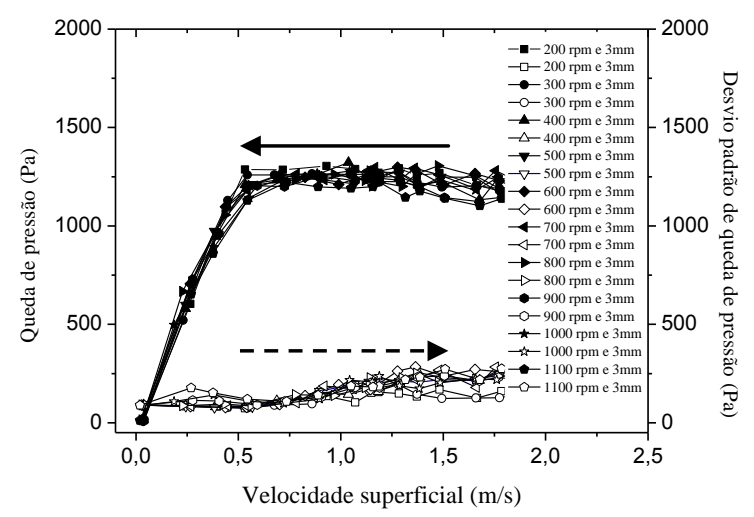

Fonte: Acervo pessoal.

Ao analisar o desvio padrão de queda de pressão verificou-se um comportamento semelhante para todas as condições, mostrando que na região de leito fixo, os valores do desvio padrão eram bem próximos de zero. A partir do momento que foi alcançada a fluidização incipiente, os valores do desvio padrão começaram a aumentar até ficarem constantes. Porém, mesmo os valores de $\delta p$ estando muito próximos, foi possível constatar que o aumento da frequência fez com que os $\delta p$ tivessem um pequeno aumento, mostrando que ao aumentar $\mathrm{f}$, as oscilações da $\Delta \mathrm{p}$ no interior do leito ficaram um pouco maiores.

Isso pode estar relacionado com as forças presente durante a fluidização das partículas, força inercial promovida pelo pleno do leito e a força de araste do fluido. Como na região de leito fixo a velocidade do fluido é sempre muito pequena, a força inercial, então, passa a ser dominante. Por se tratar de uma amplitude pequena, esta força que foi desenvolvida pelo pleno pode ser considerada baixa, uma vez que o deslocamento das esferas de vidro foi pequeno no interior da câmara de secagem, não influenciando na mudança da estrutural 
do leito particulado e nas oscilações de queda de pressão. Quando a fluidização incipiente foi atingida no processo, observou-se, para algumas frequências, que as oscilações de queda de pressão começaram a aumentar até ficarem constantes, à medida que aumentouse a velocidade superficial do ar. Isso pode estar relacionado com a força de arraste que começou a dominar a dinâmica do leito e auxiliar no deslocamento das partículas, intensificando assim o número de colições entre partícula-partícula, a partícula-parede e partícula-pleno, aumentando as oscilações de queda de pressão com o aumento da velocidade do ar.

Ao verificar os valores da velocidade de mínima fluidização obtidos nos ensaios, observou-se que à medida que aumentou a frequência da agitação, a variação da velocidade de mínima fluidização não foi tão expressiva, variando de 0,6 a $0,8 \mathrm{~m} / \mathrm{s}$.

A Figura 6 apresenta as curvas de $\Delta \mathrm{p}$ e do $\delta \mathrm{p}$ em função da velocidade superficial do ar, para as partículas com $1,19 \mathrm{~mm}$ de diâmetro e $\mathrm{A}=0,015 \mathrm{~m}$ combinada com diferentes frequências.

Figura 6- Queda de pressão e desvio padrão de queda de pressão em função da velocidade superficial do ar, para as esferas de vidro com $1,19 \mathrm{~mm}$ e $\mathrm{A}=0,015 \mathrm{~m}$ combinada com diferentes frequências.

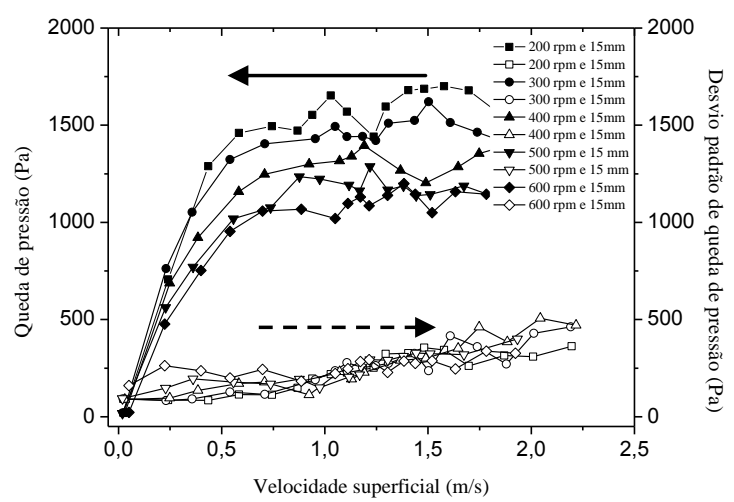

Fonte: Acervo pessoal.
Nesta figura, foi verificado com mais clareza a influência que o aumento da frequência exerceu na queda de pressão do leito, ao aumentar a amplitude de agitação, pois os valores de queda de pressão tiveram uma expressiva redução. Como a queda de pressão é influenciada pela porosidade do leito, ao aumentar o f, aumentou-se a porosidade do leito de partículas e facilitou que o fluido percole no interior do material inerte, diminuindo assim o $\Delta \mathrm{p}$.

Comportamentos semelhantes foram encontrados por autores como Meili, Daleffe e Freire (2012), ao trabalharem com partículas inferiores a um $1 \mathrm{~mm}$ de diâmetro, onde verificaram o mesmo comportamento da queda de pressão quando a amplitude foi igual a $0,014 \mathrm{~m}$ combinada com diferentes frequências.

Ao verificar $\delta \mathrm{p}$ obtidos, constatou-se que para as frequências de 200 e $300 \mathrm{rpm}$, as oscilações na queda de pressão foram mais acentuadas apenas na região de leito fluidizado. Comportamento semelhante ao encontrado na figura anterior. Para as outras condições, as oscilações de queda de pressão iniciaram na região leito fixo. Isso pode estar relacionado com a força inercial presente durante a agitação desenvolvida nestas condições, uma vez que a força inercial é responsável pela impulsão das partículas durante as colisões. Ao considerar que as esferas de vidro apresentam uma colisão elástica, a força exercida no pleno fez com que as partículas se deslocassem com maior velocidade. Com o aumento da frequência de agitação, aumentou-se o número de colisões do pleno com as esferas de vidro, tornando maior o número de colisões entre as partículas e partículas-parede, promovendo assim uma movimentação mais heterogênea das esferas de vidro e uma oscilação significativa na queda de pressão, mesmo na região de leito fixo.

A Figura 7 apresenta algumas curvas de $\Delta \mathrm{p}$ e do $\delta \mathrm{p}$ em função da velocidade 
superficial do ar, para as esferas com 2,19mm de diâmetro e amplitude de agitação igual a $0,003 \mathrm{~m}$. Nesta figura foi verificado um comportamento semelhante ao encontrado para as esferas de vidro com diâmetro de $1,19 \mathrm{~mm}$, mostrando que para as diferentes frequências trabalhadas, os valores obtidos de queda de pressão máxima foram bem próximos. Podendo considerar que, para esta amplitude e tamanho de esfera, as porosidades e as estruturas desenvolvidas no leito particulado, nas diferentes frequências, foram semelhantes e por isso as quedas de pressão não tiveram uma variação tão discrepante. Diante disso, não foram apresentados na figura todos os resultados obtidos para esta amplitude. Ao analisar a velocidade de mínima fluidização, observou-se o comportamento esperado, em que o aumento de $\Gamma$, à medida que aumentou a frequência, promoveu um aumento pouco expressivo, variando de 0,9 a $1,1 \mathrm{~m} / \mathrm{s}$.

Figura 7- Queda de pressão e desvio padrão de queda de pressão em função da velocidade superficial do ar, para as esferas de vidro com $2,19 \mathrm{~mm}$ de diâmetro e $\mathrm{A}=0,003 \mathrm{~m}$ combinada com diferentes frequências.

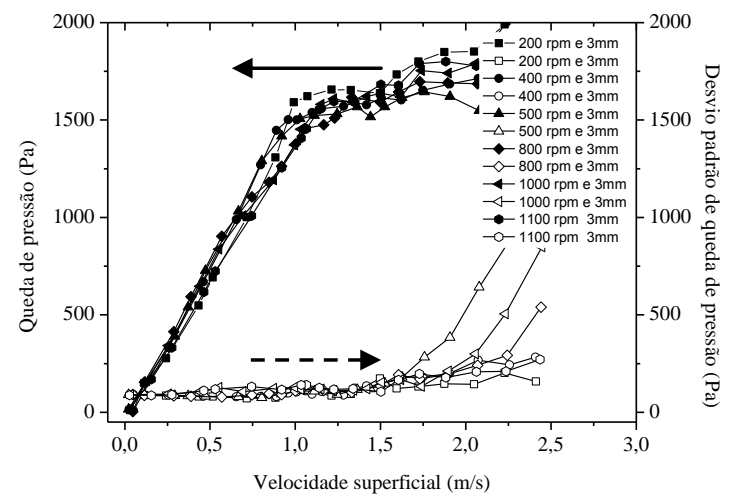

Fonte: Acervo pessoal.

Resultado semelhante também foi encontrado por Daleffe (2005), que estudou o comportamento fluidodinâmico de um leito vibrofluidizado com esferas de vidro de 2,19mm de diâmetro, utilizando a mesma amplitude combinada com diferentes frequências, e também nenhuma tendência foi encontrada para caracterizar o leito.

Ao analisar o desvio padrão de queda de pressão verificou-se que mesmo se tratando de uma esfera maior, observou um comportamento bem semelhante dos $\delta p$ encontrados para as esferas menores. No entanto, constatou-se que a partir de $2,0 \mathrm{~m} / \mathrm{s}$, os $\delta \mathrm{p}$ foram mais acentuados para algumas condições (200, 500, 800 e 1000 rpm). Como foram condições aleatórias, não sendo observada uma tendência clara de que o aumento da frequência promoveu um aumento mais acentuado das oscilações de queda de pressão, esta questão ainda será estudada mais a fundo.

Figura 8- Queda de pressão e desvio padrão de queda de pressão em função da velocidade superficial do ar, para as esferas de vidro com $2,19 \mathrm{~mm}$ e $\mathrm{A}=0,015 \mathrm{~m}$ combinada com diferentes frequências.

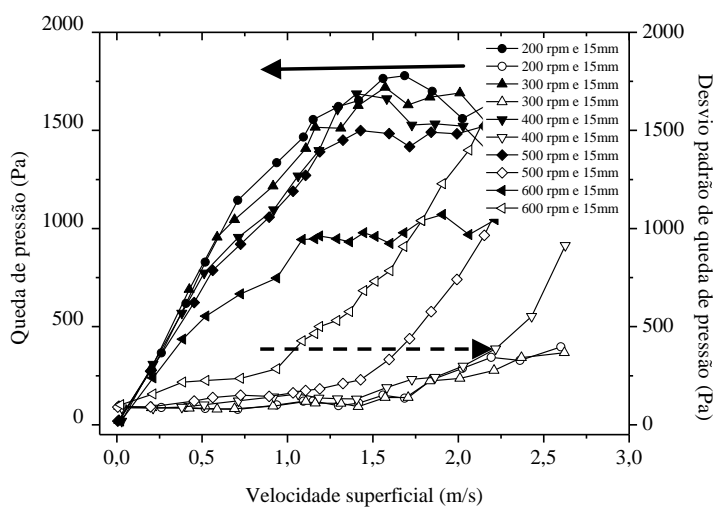

Fonte: Acervo Pessoal.

A Figura 8 apresenta as curvas de $\Delta \mathrm{p}$ e do $\delta \mathrm{p}$ em função da velocidade superficial do ar, para as esferas com $2,19 \mathrm{~mm}$ de diâmetro e amplitude de agitação igual a $0,015 \mathrm{~m}$.

Os resultados mostrados nesta figura apresentaram semelhanças com os dados encontrados pelas esferas de vidro de menor diâmetro, em que nesta amplitude a dinâmica do leito foi expressivamente mais influenciada pela frequência de vibração. 
Logo, ao aumentar a amplitude de agitação e, em conjunto, aumentando a frequência, a porosidade do leito particulado foi tornandose maior, facilitando a passagem do ar através das esferas de vidro, promovendo assim uma tendência de redução da $\Delta \mathrm{p}$. Ao analisar a velocidade de mínima fluidização, observouse novamente que esta variável não apresentou um significativo aumento com o aumento da frequência, obtendo valores que variaram de 1,15 a $1,25 \mathrm{~m} / \mathrm{s}$.

As curvas de $\delta p$ do leito com esta amplitude mostraram na região de leito fixo, apenas que a condição de $600 \mathrm{rpm}$ apresentou oscilações significativas de queda de pressão. Para o restante das condições, as oscilações de queda de pressão começaram na região de fluidização incipiente. Como esta esfera de vidro apresenta uma massa maior de partícula, a força inercial que foi desenvolvida pelo pleno na região de leito fixo, com as frequências menores que $600 \mathrm{rpm}$, não foram suficientes para desenvolver uma movimentação heterogênea das esferas de vidro que pudessem produzir oscilações significativas de $\Delta \mathrm{p}$. A partir do momento que a força de arraste do fluido começou a ser significante no processo, iniciou-se então uma movimentação heterogênea bem expressiva do material inerte, devido o aumento do número de colisões entre, partícula-partícula, partícula-parede e partícula-pleno que resultaram em oscilações bem significativas da queda de pressão.

\section{CONCLUSÕES}

Neste trabalho foi observado que as curvas de $\Delta \mathrm{p}$ do leito vibrofluidizado apresentaram um comportamento clássico em que ocorre a amortização da transição do leito fixo para o leito fluidizado. Para a amplitude de $0,003 \mathrm{~m}$, não fica evidente a influência da frequência nos valores de queda de pressão e na velocidade de mínima fluidização durante o processo, mesmo o leito operando com diâmetros diferentes de esferas. Nos resultados encontrados da amplitude de $0,015 \mathrm{~m}$, para as duas esferas de vidro, pode constatar que os valores de queda de pressão apresentaram uma redução bem expressiva, com o aumento da frequência. Porém a $U_{\mathrm{mf}}$, não sofreu tanta influência da frequência durante o processo. Além de mostrar o que vem sendo dito na literatura, que $\Gamma$ não deve ser apresentado unicamente como parâmetros para caracterizar o leito, devendo ser informado sempre as variáveis A e f.

\section{NOMENCLATURA}

$\begin{array}{lllll}A & \begin{array}{l}\text { Amplitude } \\ d_{p}\end{array} & \begin{array}{l}\text { Diâmetro médio da } \\ \text { partícula }\end{array} & {[\mathrm{L}]} \\ f & \begin{array}{l}\text { Frequência } \\ g\end{array} & \begin{array}{l}\text { Aceleração da gravidade } \\ \Delta P\end{array} & \begin{array}{l}\text { Queda de pressão no leito } \\ {[\mathrm{Hz}]}\end{array} \\ \delta p & \begin{array}{l}\text { Desvio padrão de queda de } \\ \text { pressão }\end{array} & {\left[\mathrm{ML}^{-1} \mathrm{~T}^{-2} \mathrm{~T}^{-2}\right]} \\ U_{m f} & \begin{array}{l}\text { Velocidade mínima de } \\ \text { fluidização }\end{array} & {\left[\mathrm{LT}^{-1}\right]} \\ U_{s} & \begin{array}{l}\text { Velocidade superficial do } \\ \text { ar }\end{array} & {\left[\mathrm{LT}^{-1}\right]}\end{array}$

Letras gregas

Г Número do adimensional [-] de vibração

\section{REFERÊNCIAS}

BRATU, E.; JINESCU, G.I. Effect of Vertical Vibrations on the Pressure Drop in a Fluidized Layer. Brit. Chem. Eng., v.16, n.8, p.691-695,1971.

COSTA, S. S.; MORIS, V.A.S.; ROCHA, S.C.S. Influence of process variables on granulation of microcrystalline cellulose in vibrofluidized bed. Powder Technol., v. 207, p. 454-460, 2011. 
DALEFFE, R. V. Contribuições para a análise do comportamento fluidodinâmico de um leito vibrofluidizado. Tese (Doutorado em Engenharia Química) Universidade Federal de São Carlos, São Carlos, 2005.

DALEFFE, R. V.; FERREIRA, M. C.; FREIRE, J. T. Drying of Paste in VibroFluidized Bed: Effects of the Amplitude and Frequency of Vibration. Drying Tecnnol. V. 23. p. 1765-1781. 2005.

DALEFFE, R. V.; FERREIRA, M.C.; FREIRE, J.T. Effects of binary particle size Distribution on the fluid dynamic behavior of fluidized, vibrated and vibrofluidized beds. Braz. J. Chem. Eng., v.25, n.1, p. 83-94, 2008.

FREIRE, J. T.; FERREIRA, M. C.; FREIRE, F. B. NASCIMENTO, B. S. Review on Paste Drying with inert Particles as Support Medium. Drying Technol., v.30.p.330-341. 2012.

GARIM, M. M., Estudos dos coeficientes de transferência de massa em um leito fluidizado e vibro-fluidizado. Tese (Doutorado em Engenharia Química) Universidade Federal de São Carlos, São Carlos 1998.

GELDART, D. Types of Gas Fluidization. Powder Technol.,v. 7,p. 285-292, 1973.

GUPTA, R.; MUJUMDAR, A. S. Aerodynamics of a Vibrated Fluid Bed. The Canad. J. of Chem. Eng., v. 58, p. 332-338, 1980.

KUNII, D.; LEVENSPIEL, O. Fluidization Engineering. Academic Press, New York. p. 491.2 ed. 1991
MEILI, L. Contribuições ao Estudo da fluidodinâmica e da secagem de pastas em leito fluidizado e vibrofluidizado. 2009. 218 f. Tese (Doutorado em Enegenharia Química) - Universidade Federal de São Carlos, São Carlos, 2009.

MEILI, L.; DALEFFE，R.V.; FERREIRA, M. C. FREIRE, J.T. Analysis of the Influence of Dimensionless Vibration Number on the Drying of Pastes in Vibrofluidized Beds. Drying Technol., v. 28, p.402-411, 2010.

MEILI, L.; DALEFFE, R.V.; FREIRE, J.T. Fluid Dynamics of Fluidized and Vibrofluidized Bed Operating with Geldart C Particles. Chem. Eng. Technol., v. 35, n. 9, p. 1649-1656, 2012.

NUNES, J.F.; ALCANTARA, F.C.A.; SILVA-MORIS, V.A.S.; ROCHA, S.C.S. Fluid dynamics and coating of sodium bicarbonate in a vibrofluidized bed. Chem. Eng. and Process., v. 52, p. 34-40. 2012.

SEVILLE， J.P.K.; WILLETT, C.D.; KNIGHT. P.C. Interparticle forces in fluidization: a review. Powder Technol. v. 113, p.261-268. 2000.

SILVA-MORIS， V.A.; ROCHA， S.C.S. Vibrofluidized Bed Drying of Adipic Acid. Drying Technol. v. 24, p. 303-313, 2006.

\section{AGRADECIMENTOS}

Os autores agradecem ao $\mathrm{CNPq}$ pelo auxílio financeiro. 